\title{
High-frequency dynamics of glass-forming polybutadiene
}

\author{
D. Fioretto, ${ }^{1}$ U. Buchenau, ${ }^{2}$ L. Comez, ${ }^{1}$ A. Sokolov,${ }^{3}$ C. Masciovecchio, ${ }^{4}$ A. Mermet, ${ }^{4}$ G. Ruocco, ${ }^{5}$ F. Sette, ${ }^{4}$ L. Willner,${ }^{2}$ \\ B. Frick, ${ }^{6}$ D. Richter, ${ }^{2}$ and L. Verdini ${ }^{1}$ \\ ${ }^{1}$ Dipartimento di Fisica and INFM, Universitá di Perugia, I-06100 Perugia, Italy \\ ${ }^{2}$ Institut für Festkörperforschung, Forschungszentrum Jülich, D-52425 Jülich, Federal Republic of Germany \\ ${ }^{3}$ Department of Polymer Science, University of Akron, Akron Ohio 44325-3909 \\ ${ }^{4}$ European Synchrotron Radiation Facility, Boîte Postale 220 F-38043 Grenoble, Cedex, France \\ ${ }^{5}$ Dipartimento di Fisica and INFM, Università di L'Aquila, I-67100 L'Aquila, Italy \\ ${ }^{6}$ Institut Laue-Langevin, Boîte Postale 156, F-38042 Grenoble Cedex, France
}

(Received 21 October 1998)

\begin{abstract}
Inelastic x-ray measurements of polybutadiene are reported, performed over a wide temperature range covering both the glass and the liquid phase. At each temperature, the frequency position $\Omega$ and the width $\Gamma$ of the inelastic peaks of the spectra have been obtained for different values of the scattering vector $Q$. A linear behavior of $\Omega(Q)$ for $Q<4 \mathrm{~nm}^{-1}$ has been revealed, allowing the determination of the unrelaxed sound velocity $v_{\infty}$. Consistently with the results obtained in different glass-forming systems, the $Q$ dependence of $\Gamma$ is well represented by a $Q^{2}$ law. For $Q>5 \mathrm{~nm}^{-1}$ the values of $\Gamma$ overtake those of $\Omega$ and the acousticlike excitations progressively loose their propagative nature. In the glass, $v_{\infty}(T)$ compares well with previous Brillouin Light Scattering (BLS) determinations, while in the liquid the BLS sound velocity shows a steeper temperature dependence related to the structural relaxation. The temperature behavior of the nonergodicity factor has been derived both from $v_{o}$ and $v_{\infty}$ (in the liquid phase) and from the ratio between elastic and inelastic intensities of inelastic $\mathrm{x}$-ray scattering spectra (in the whole investigated temperature range). Both temperature and $Q$ behavior of this quantity might be consistently interpreted in the framework of the mode coupling theory. [S1063-651X(99)10004-7]
\end{abstract}

PACS number(s): 64.70.Pf, 61.10.Eq, 63.50.+x, 78.70.Ck

\section{INTRODUCTION}

The dynamics of polymeric glass forming systems, as that of simple liquids, is affected by different relaxation processes. Among these, the slowing down of the structural $(\alpha-)$ relaxation is responsible for the glass transition. In recent years theoretical models have been developed that give detailed description of the dynamics of supercooled systems, in the temperature region above the glass-transition temperature $T_{g}$, such as the mode coupling theory (MCT) [1]. This theory predicts the existence of a dynamic transition located at $T_{c}>T_{g}$, similar to that found in the study of spin glasses [2], where the transition from ergodic to nonergodic behavior occurs. A quantitative signature of this dynamic transition is expected in the Debye-Waller factor $f_{Q}$, which is expected to show a square-root cusp in its temperature behavior at $T_{c}$ [1]. The experimental evidence of the existence of such a cusp has been obtained in 1,4polybutadiene (PB), by inelastic neutron scattering (INS) measurements, in the $Q$ range between the first and second maximum of the static structure factor, i.e., between about 14 and $27 \mathrm{~nm}^{-1}$ [3-6].

This investigation can be extended to lower values of $Q$ by means of inelastic x-ray scattering (IXS), Brillouin light scattering (BLS) and ultrasonic measurements (US). In particular, in the $Q \rightarrow 0$ limit, $f_{0}$ can be obtained as $f_{0}=1$ $-v_{0}^{2} / v_{\infty}^{2}$ [1], where $v_{0}$ and $v_{\infty}$ are the relaxed and unrelaxed sound velocities, respectively. The relaxed condition $(\omega$ $\ll 1 / \tau_{\alpha}$ ) can be met by low-frequency spectroscopies, like US; thus, $v_{0}$ can be easily measured in a large temperature range, from the liquid phase down nearly to the calorimetric glass transition temperature $\left(T_{g}\right)$. The determination of $v_{\infty}(T)$ is much more difficult. BLS methods allow us to measure the dynamic structure factor $S(Q, \omega)$ up to $Q$ $\approx 0.04 \mathrm{~nm}^{-1}$, and therefore to detect the sound wave speed up to about $10 \mathrm{GHz}$, which give the values of $v_{\infty}(T)$ only in the glass and in the deeply undercooled liquid. BLS has been also used for determining the values of $v_{\infty}(T)$ at temperatures higher than $T_{g}$ by means of a spectral shape analysis $[7,8]$. However, in this analysis one faces the problem of the large number of statistically correlated parameters, which can give unreliable values of $v_{\infty}(T)$ and thus, in turn, of $f_{0}$. With this respect, an independent determination of $v_{\infty}(T)$ would be strongly desirable

The recent development of the IXS technique allows one to study $S(Q, \omega)$ in a $(Q, \omega)$ regime accessible neither to BLS nor to INS (due to kinematic limitations). This gives the possibility of measuring the dispersion relation of acoustic excitations $\Omega(Q)$ up to frequencies where the condition $\Omega(Q) \gg 1 / \tau_{\alpha}$ is truly verified also in the liquid state. The quantity $v_{\infty}(T)$ is then derived as the $Q \rightarrow 0$ limit of $\Omega(Q, T)$ [9]. Moreover, IXS allows one to determine $f_{Q}$ in the intermediate $Q$ range by the ratio of elastic to total scattered intensity $f_{Q}=I_{e l} / I_{\text {tot }}[9]$.

In the present paper we report an IXS study of deuterated $\mathrm{PB}$, in the temperature range $50-320 \mathrm{~K}$, i.e., from the glass to the viscous liquid phases, and in the $Q$ range $1-10 \mathrm{~nm}^{-1}$. From the analysis of the spectra it has been possible to obtain the temperature dependence of both $v_{\infty}$ and the DebyeWaller factor $f_{Q}$ at different $Q$ values, in the whole tempera- 
ture range. A qualitative agreement has been found with the predictions of mode coupling theory. In particular, the leveling off of $f_{Q}$ at high temperatures and its slight increase with $Q$ could possibly be explained by this theory.

\section{EXPERIMENT AND RESULTS}

The experiment has been carried out at the very high energy resolution IXS beamline (BL21-ID16) at the European Synchrotron Radiation Facility. The incident $x$-ray beam is obtained by the combination of a cryogenically cooled $\mathrm{Si}(111)$ double crystal monochromator and a very high energy resolution back-scattering monochromator operating at the $\operatorname{Si}\left(\begin{array}{lll}11 & 11 & 11\end{array}\right)$ reflection [10]. The scattered photons are collected by a spherical silicon crystal analyzer, also operating at the $\mathrm{Si}\left(\begin{array}{lll}11 & 11 & 11\end{array}\right)$ back reflection [11]. The monochromatic beam has an energy of $\approx 21,478 \mathrm{eV}$ and an intensity of $2 \times 10^{8}$ photons/s. The total energy resolution, determined from the elastic scattering of a Plexiglas sample at the maximum of its static structure factor, is $1.5-\mathrm{meV}$ full width half maximum. The momentum transfer, $Q=2 k_{0} \sin \left(\theta_{s} / 2\right), k_{0}$ being the wave vector of the incident photon and $\theta_{s}$ being the scattering angle, is selected by five different detectors. The total $Q$ resolution has been set to $0.2(0.4) \mathrm{nm}^{-1}$ for $Q \leqslant(>) 2 \mathrm{~nm}^{-1}$. Energy scans are done by varying the relative temperature between the monochromator and the analyzer crystals. Each scan has taken about $20^{\prime}$, and the collected data have been normalized to the intensity of the incident beam.

A deuterated 1,4-polybutadiene sample with molecular weight 25.000 was prepared by anionic polymerization. The cell length was chosen to be comparable to the x-ray photoabsorption length, and multiple scattering was negligible. No spectral changes were observed among different temperature scans at the same temperature, or approaching the selected temperature from either above or below.

Inelastic $\mathrm{x}$-ray measurements of polybutadiene were performed at 12 different temperatures in the range 50-320 K. At each temperature, five different $Q$ values have been typically detected, i.e., $1,2,4,7$, and $10 \mathrm{~nm}^{-1}$. To better reconstruct the dispersion curves, other sets of $Q$ s were measured at two significative temperatures. In particular, 3, 6, 9, 12, and $15 \mathrm{~nm}^{-1}$ were also measured at 294 and $140 \mathrm{~K}$ and, in addition, $1.5,4.5,7.5$, and $10.5 \mathrm{~nm}^{-1}$ spectra were taken at $T=140 \mathrm{~K}$.

The IXS spectra of PB at $Q=2 \mathrm{~nm}^{-1}$ for different values of the temperature are shown in Fig. 1. The spectra show three main components: a quasielastic peak and two inelastic peaks. The frequency position of the inelastic peaks clearly shows a temperature dependence, which is more pronunced in the high temperature regime.

In order to extract the relevant spectroscopic features of the spectra, such as the frequency position and the width of the inelastic features, and the intensity of both elastic and inelastic features, a fitting procedure was used. It was based on the convolution of the experimentally determined resolution function with a model function $S(Q, \omega)$. The central line was represented by a Lorentzian and the inelastic peaks by a DHO (damped harmonic oscillator) [12]:

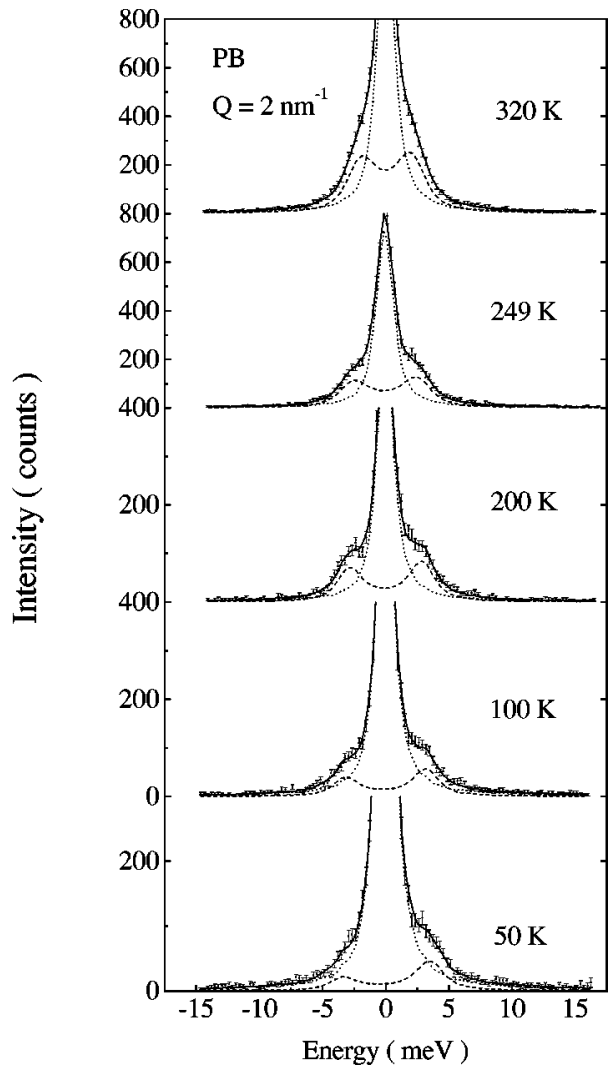

FIG. 1. IXS spectra of polybutadiene at $Q=2 \mathrm{~nm}^{-1}$, at the indicated temperatures. The data $(\bigcirc)$, shown with the error bars, are superimposed to the fit (solid line) as explained in the text. The dotted (dashed) lines represent the quasielastic (inelastic) contributions to the total fits.

$$
\begin{aligned}
S(Q, \omega)= & \frac{I_{e l}(Q)}{\pi} \frac{\Gamma_{c}(Q)}{\omega^{2}+\Gamma_{c}(Q)^{2}}+\frac{I(Q)}{\pi}\left[(n(\omega)+1) \frac{\hbar \omega}{k T}\right] \\
& \times \frac{\Gamma(Q) \Omega(Q)^{2}}{\left(\Omega(Q)^{2}-\omega^{2}\right)^{2}+\Gamma(Q)^{2} \omega^{2}} .
\end{aligned}
$$

Here, $I_{e l}(Q)$ and $I(Q)$ are the intensities of the central peak and of the inelastic contribution $\left(I_{t o t}(Q)=I_{e l}(Q)+I(Q)\right)$, $\Omega(Q), \Gamma(Q)$, and $\Gamma_{c}(Q)$ refer to the central frequency and to the energy widths of the side and central lines, and $n(\omega)$ is the Bose factor. The convolution of $S(Q, \omega)$ with the instrument resolution function is fitted to the spectrum using standard $\chi^{2}$ minimization. The arbitrary choice of the DHO is made because it contains the basic features of the inelastic part of the $S(Q, \omega)$ of a disordered system, and allows the determination of the main spectroscopic parameters, as demonstrated on other real and simulated systems [13-15].

The individual contributions of elastic and inelastic components to the spectra are shown by the dotted and dashed lines in Fig. 1.

Figure 2 shows the typical $Q$ dependence of the IXS spectra of PB at $T=140 \mathrm{~K}$. It can be seen that the energy position of the inelastic peaks increases and the width becomes progressively broader with increasing $Q$. This marked $Q$ dispersion of the inelastic peaks is qualitatively the same in the whole temperature range investigated here and is very similar to that previously found in other glass forming systems 


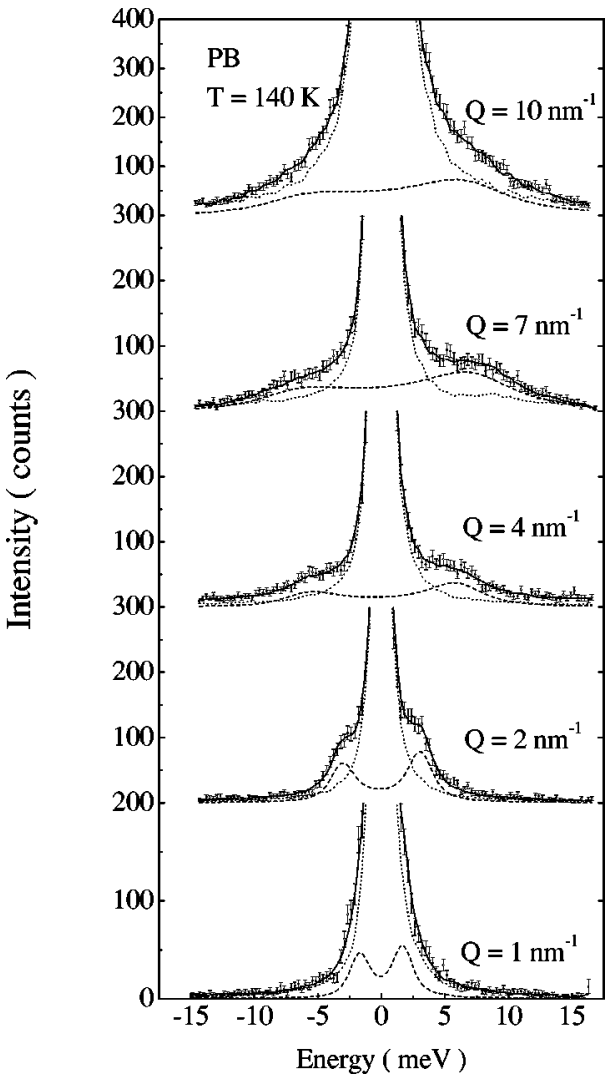

FIG. 2. IXS spectra of polybutadiene at $140 \mathrm{~K}$, at the indicated $Q$ values. A marked $Q$ dispersion and broadening of the inelastic peaks (dashed lines) can be seen.

$[14,16]$. This behavior suggests the persistence of the acousticlike nature of the collective dynamics of PB up to these high frequencies, as better evidenced in Fig. 3.

In Fig. 3 the frequency position and the width of the inelastic peaks of the spectra taken at $140 \mathrm{~K}$ for different values of the scattering vector are reported. Similar trends for $\Omega(Q)$ and $\Gamma(Q)$ have been found in the whole investigated temperature range. A linear behavior of $\Omega$ up to $Q$ $\approx 4 \mathrm{~nm}^{-1}$ is visible in the figure, the slope giving the value of the sound velocity $v_{I X S}=2.64 \mathrm{~km} / \mathrm{s}$. A different $Q$ dependence is found for the width $\Gamma$ which, for $Q<5 \mathrm{~nm}^{-1}$ is

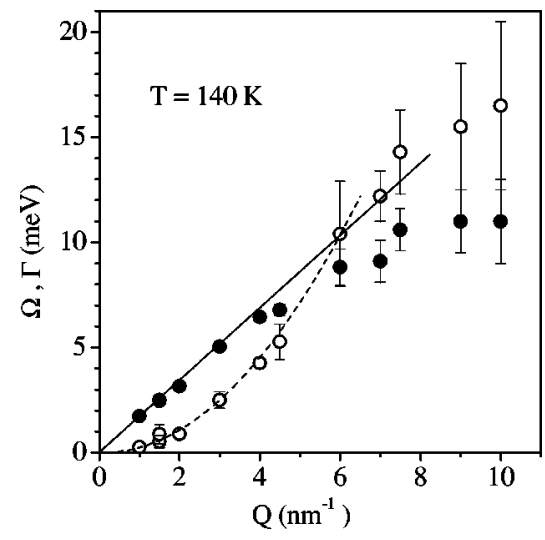

FIG. 3. Frequency position $(\Omega, \bullet)$ and width $(\Gamma, \bigcirc)$ of the inelastic peaks of IXS spectra of polybutadiene at $140 \mathrm{~K}$. The solid and dashed lines correspond to the fit of the linear $Q$ dispersion of $\Omega$ and of the $Q^{2}$ behavior of $\Gamma$, respectively.

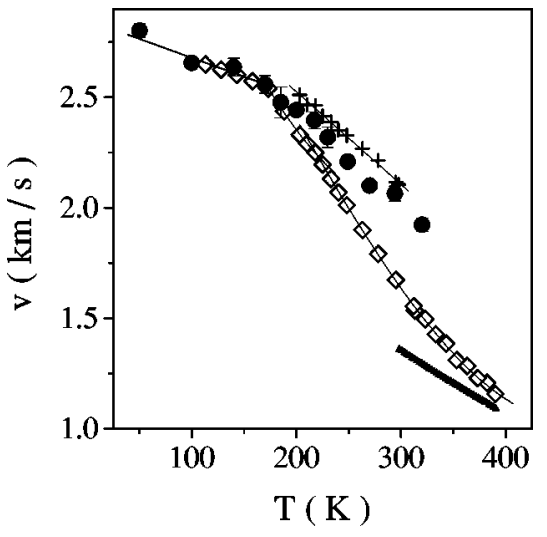

FIG. 4. Temperature behavior of the apparent sound velocities of polybutadiene obtained by IXS $(\mathbf{O})$, Brillouin light scattering peaks $(\diamond)$, and ultrasonic measurements $(\triangle)$. The values of $v_{\infty}$ obtained by fitting Brillouin light spectra $(+)$ are also reported for sake of comparison.

well represented by a $Q^{2}$ law. It resembles a hydrodynamic behavior, as already observed in a number of glass forming systems by means of both IXS $[14,17,9]$ and BLS [18] techniques. It is worth noting that despite the large number of experimental evidences, this $Q^{2}$ law still deserves a convincing theoretical explanation.

In Fig. 4 the temperature dependence of $v_{I X S}$ is reported together with that obtained by BLS and US measurements previously performed on a nondeuterated sample [7]. The $v_{I X S}$ data have been obtained by a linear fit of $\Omega(Q)$ in the low $Q$ limit, as previously discussed for the case $T=140 \mathrm{~K}$. The BLS and US data are rescaled to correct for the differencies in mass density between deuterated and nondeuterated samples. In this plot, BLS data show a linear temperature behavior in the glassy state, where $v_{B L S} \approx v_{\infty}$. For increasing temperature a change of the slope is visible not far from $T_{g}$, where a more pronunced temperature dispersion starts to occur. At the highest temperatures, the values of $v_{B L S}$ progressively approach those of $v_{0}$ as obtained by ultrasonic measurements. In the glass, i.e., for $T<180 \mathrm{~K}$ we find good agreement between BLS and IXS determinations of $v_{\infty}$. This gives us confidence that the procedure used to extract $v_{\infty}$ from the IXS experimental data is meaningful, allowing the determination of $v_{\infty}$ also in the liquid, i.e., in a temperature range not directly accessible before. It is worth comparing the $v_{\infty}$ values derived here in the liquid phase with those determined by the line-shape analysis of the BLS spectra. In Ref. [7], some of us studied the temperature dependence of the BLS spectra of hydrogenated $\mathrm{PB}$, representing the isotropic spectral component with a model based on a single Kohlrausch Williams Watts (KWW) memory function:

$$
m_{Q}(t)=\Delta_{Q}^{2} \exp \left[-(t / \tau)^{\beta}\right]
$$

where $\Delta_{Q}^{2}=\left(v_{\infty}^{2}-v_{0}^{2}\right) Q^{2}$ is the relaxation strength. In this approach the infinite frequency sound velocity is a fitting parameter. The values of $v_{\infty}$ derived from BLS definitely lie above the IXS determination. This result has been also found in two other fragile glass formers, specifically in metatricresylphosphate $[19,20]$ and propylene carbonate $[21,22]$, where the BLS determination of $v_{\infty}$ is always overestimated. 
One possible explanation of such a discrepancy can be ascribed to the procedure used to extract $v_{\infty}$ from the BLS spectra. Indeed, $v_{\infty}$ is usually obtained from the value of $v_{0}$, obtained by US measurements, and that of $\Delta^{2}$, which is a free fitting parameter, giving values that strongly depend on the specific model chosen for the memory function. If, for instance, two relaxation processes are active in the investigated temperature region, the use of a single relaxation memory function can lead to an overstimated value of $\Delta^{2}$ [23].

A second, more subtle, explanation can be found in the following argument. The value of $\Delta^{2}$ derived from fitting BLS spectra corresponds to the initial $(t=0)$ value of the memory function $m(t)$ of Eq. (2.2). Due to the limited frequency windows accessible to BLS, the parameters of the memory function $\left(\Delta^{2}, \beta\right.$, and $\left.\tau\right)$ are determined at finite values of time $\left(t \approx 1 / \omega_{B} \approx 10 \mathrm{ps}\right)$. Therefore, the value of $\Delta^{2}$ that comes out from the fitting procedure can be thought to be an "extrapolation" at $t=0$ of $m(t)$. This function, as written in Eq. (2.2), is characterized by an unphysical cusp behavior at $t=0$. Different from that, a "true" memory function, $m_{t}(t)$ must be even in time with all the derivative continues at $t=0$, i.e., it must not show cusp at $t=0$. It is easy to see that, at short time, in order to avoid the cusp, $m_{t}(t)$ must go to a constant level with $m_{t}(t)<m(t)$. Therefore, also the relation $\Delta_{t}^{2}<\Delta^{2}$ holds, and, consequently, the $v_{\infty}$ values derived from the fit are overestimated. This problem can become more and more important for increasing temperature: whenever the time accessed by BLS becomes longer than the typical relaxation time $\left(1 / \omega_{B} \gg \tau\right)$, one can obtain unphysical values of $v_{\infty}$ (see, for instance, Fig. 8 of Ref. [26]).

It is important to emphasize that the IXS determination of $v_{\infty}$ can be used as independent information in the analysis of the BLS spectra, therefore reducing the number of freefitting parameters and allowing a better determination of the relaxation time $\tau$.

The temperature behavior of the linewidth $\Gamma$ of IXS inelastic spectra is reported in Fig. 5 for different values of $Q$. A linear fit of the temperature dependence of $\Gamma$ has been performed, corresponding to the dotted lines of Fig. 5. The slope of this line is very weak and, within the experimental error, no appreciable changes are visible in the whole temperature range, suggesting that the mechanism, which is responsible for broadening in the glassy phase, continues to dominate also for temperatures higher than $T_{g}$. This result can be explained by the fact that, at the probed frequencies, the unrelaxed condition $\omega \tau \gg 1$ is satisfied in the whole investigated temperature range [34] and, therefore, the sound waves feel the system as "frozen." In this regime the broadening of the excitations peaks in the $S(Q, \omega)$ is due to the disordered molecular structure rather than to truly dynamics processes.

From these arguments and from the results of Fig. 5, we do not expect any influence of the structural and secondary relaxation process on the measured speed of sound $v(Q)$, thus giving further confidence to the values of $v_{\infty}$ previously obtained by IXS spectra.

Among the predictions of the idealized MCT there is a singular behavior of the nonergodicity factor $f_{Q}$ at the criti-

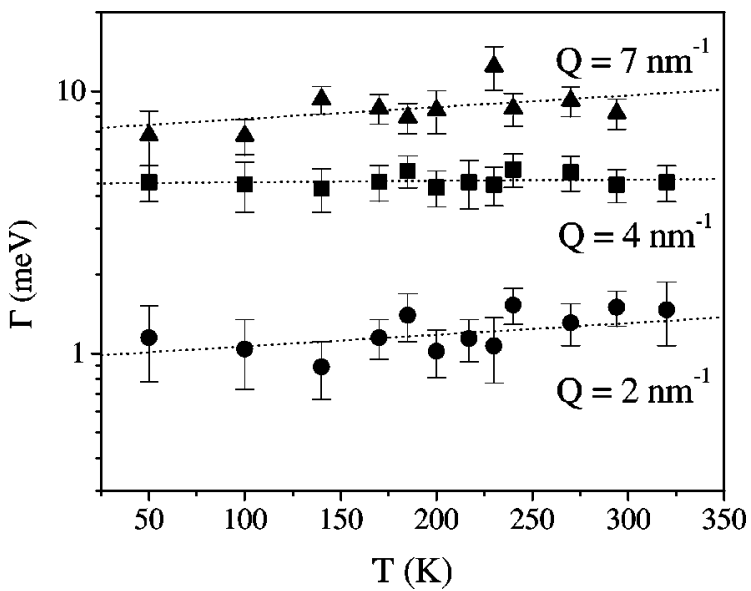

FIG. 5. Width $(\Gamma)$ of the inelastic peaks of IXS spectra of polybutadiene as a function of temperature, for different values of $Q$. The absence of appreciable variations of $\Gamma_{Q}(T)$ evidenced by the linear fits (dotted lines) suggests that the mechanism responsible for the sound attenuation in the liquid phase is still the same as that active in the glass.

cal temperature of the system which, in case of $\mathrm{PB}$, has been located at $T_{c}=216 \mathrm{~K}[3-6]$. The $f_{Q}$ factor is typically measured in two different $Q$ regimes: in the high $Q$ regime, $f_{Q}$ is determined by inelastic neutron scattering experiments both by measuring the relative area of the quasielastic peak in $S(Q, \omega)$ and by measuring the intermediate scattering function $F(Q, t)$ at short times [25]; in the low $Q$ regime, $f_{Q=0}$ is obtained from $v_{\infty}$ data resulting from fitting routines on BLS spectra $[7,8,26]$ and using the relationship $f_{0}=1$ $-\left(v_{o} / v_{\infty}\right)^{2}[27]$.

A direct access to this quantity can be now gained from IXS measurements. Thanks to the peculiar $(Q, \omega)$ spanned range, the IXS technique can be used to determine $f_{Q}$ in both limits: (i) by using the $v_{\infty}$ values presented in Fig. 4 and literature $v_{0}$ data [7], we can determine $f_{0}$ from the highest studied temperature down to $T_{g}$ (Fig. 6, diamonds), i.e., down to the lowest temperature where the measured $v_{0}$ data can be reliably extrapolated; (ii) by calculating the intensity ratio between the quasielastic peak and the total $S(Q, \omega)$ as derived from the fits, we can obtain $f_{Q}$ at $Q=2 \mathrm{~nm}^{-1}, Q$ $=4 \mathrm{~nm}^{-1}$, and $Q=7 \mathrm{~nm}^{-1}$. Although the data appear to be scattered due to low statistics, some trend in the temperature dependence can be put in evidence around the glass transition. In particular, for the $f_{Q=0}$ data one observes a substantialy constant value in the liquid, similar to what was found in the previous work where the values of $v_{\infty}$ was obtained by fitting BLS spectra. [7] In that case the value of $f_{Q=0}$ was overestimated by $\approx 5 \%$ due to the higher values of $v_{\infty}$. Concerning the higher $Q$ values, $f_{Q}$ is found to decrease for increasing temperature at low $T$ and tends to become constant above a certain temperature. The exact location of this temperature from the present data is difficult and depends very much on the way in which one extrapolates the low temperature data. Here we have choosen a linear extrapolation resulting in crossover temperatures, which are consistent with the $T_{c}$ determined by inelastic neutron scattering. Due to the limited number of points in the low temperature range, a fit with a square-root temperature dependence was not attempted. Moreover, in Fig. 6 one can observe an increase 


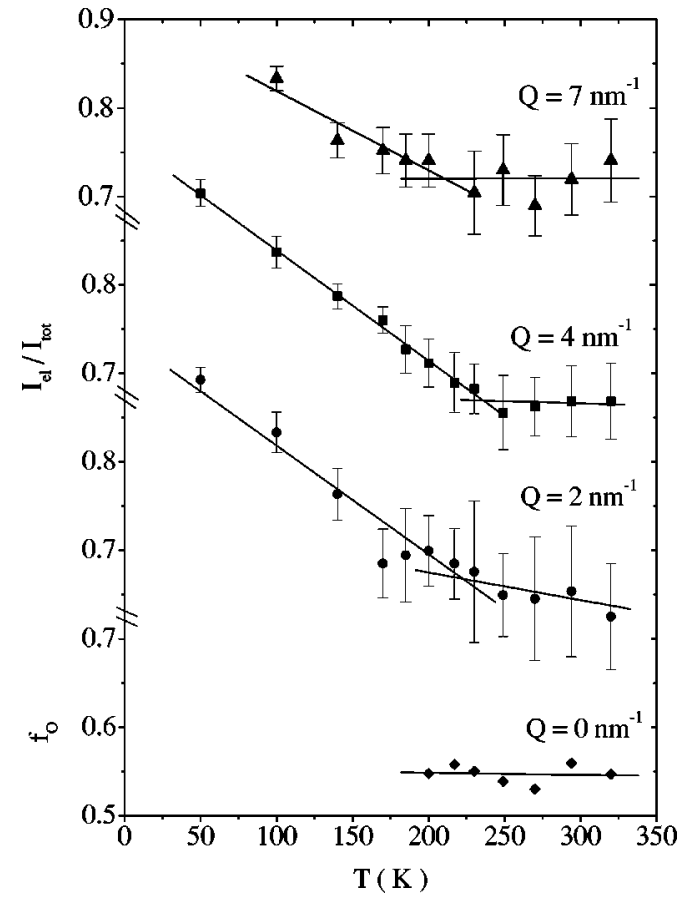

FIG. 6. Nonergodicity factor of polybutadiene calculated at $Q$ $\rightarrow 0$ from ultrasonic $\left(v_{0}\right)$ and IXS $\left(v_{\infty}\right)$ velocities as $f_{0}=1$ $-\left(v_{0} / v_{\infty}\right)^{2}$, and for different values of $Q$ from the ratio between the quasielastic intensity $\left(I_{e l}\right)$ and the total integrated intensity of IXS spectra.

with $Q$ of the values of the critical Debye-Waller factor, i.e., of $f_{Q}^{c}=f_{Q}\left(T_{c}\right)$. In particular, the value of $f_{Q}^{c}$ changes from 0.55 in the $Q \rightarrow 0$ limit to about 0.72 for $Q=7 \mathrm{~nm}^{-1}$. It is interesting to compare these results with those previously obtained by neutron spin echo and time-of-flight measurements of $f_{Q}^{c}=0.92$ close to the first maximum of the structure factor, i.e., at $Q=1.48 \AA^{-1}$, [4] and $f_{Q}^{c}=0.77$ at the first minimum of $S(Q)$, i.e., at $Q=1.88 \AA^{-1}$. [6] Combining the IXS and neutron data of $f_{Q}$, it comes out that in the reported $Q$ range, the values of $f_{Q}^{c}$ of PB oscillate in phase with the static structure factor $S(Q)$, similar to what has been recently measured in more "simple" glass-forming systems, such as colloidal suspensions of hard spheres, [28] and orthoterphenyl [29], and calculated in hard spheres, [30] Lennard-Jones spheres, [31] binary mixtures of hard spheres, [32] and, more recently, in supercooled water [33].

\section{CONCLUSIONS}

In summary, our measurements show the ability of IXS to reveal the dynamics of a glass-forming polymer in a previously unexplored $Q$ range. From the $Q \rightarrow 0$ behavior of the inelastic part of the spectra, the unrelaxed sound velocity of PB has been obtained in a wide temperature range, from the glass to the viscous liquid phase. The results favorably compares with values previously estimated by Brillouin light scattering experiments. Moreover, IXS spectra could be used to investigate the temperature behavior of the MCT nonergodicity factor $f_{Q}$ at four different $Q$ values from $\approx 0$ to $7 \mathrm{~nm}^{-1}$. A qualitative agreement has been found with the prediction of the mode coupling theory. In fact, the critical factor $f_{Q}^{c}$ as determined by IXS for PB here, when taken together with earlier neutron scattering results [4], oscillates in phase with the static structure factor, similar to what was calculated in the framework of the MCT in simple fluids. The results obtained strongly encourage a more thorough study in the vicinity of the critical temperature of this system aimed to a substantial increase of the statistics of the IXS determinations of both $v_{\infty}(T)$ and $f_{Q}(T)$. This should allow a more quantitative comparison with the prediction of MCT and, more generally, of the theories of glass-forming systems.
[1] W. Götze and L. Sjögren, Rep. Prog. Phys. 55, 241 (1992).

[2] T.R. Kirkpatrick and D. Thirumalai, Phys. Rev. B 36, 5388 (1987); L. Cugliandolo and J. Kurchan, Phys. Rev. Lett. 71, 173 (1993); S. Franz and G. Parisi, ibid. 79, 2486 (1997).

[3] B. Frick et al., Z. Phys. B 70, 73 (1988).

[4] D. Richter et al., Phys. Rev. Lett. 61, 2465 (1988).

[5] B. Frick et al., Phys. Rev. Lett. 64, 2921 (1990).

[6] D. Richter et al., Phys. Rev. Lett. 68, 71 (1992).

[7] D. Fioretto, L. Palmieri, G. Socino, and L. Verdini, Phys. Rev. B 50, 605 (1994).

[8] A. Aoudi, M.J. Lebon, C. Dreyfus, B. Strube, W. Steffen, A. Patkowski, and R.M. Pick, J. Phys.: Condens. Matter 9, 3803 (1997).

[9] G. Monaco, C. Masciovecchio, G. Ruocco, F. Sette, Phys. Rev. Lett. 80, 2161 (1998).

[10] R. Verbeni et al., J. Synchrotron Radiat. 3, 62 (1996).

[11] C. Masciovecchio et al., Nucl. Instrum. Methods Phys. Res. B 111, 181 (1996).

[12] B. Fak and B. Dorner, Institute Laue Langevin, Grenoble, France, Report No. 92FA008S, 1992.
[13] F. Sette et al., Phys. Rev. Lett. 75, 850 (1995); G. Ruocco et al., Nature (London) 379, 521 (1996); F. Sette et al., Phys. Rev. Lett. 77, 83 (1996).

[14] C. Masciovecchio et al., Phys. Rev. Lett. 76, 3356 (1996); P. Benassi et al., ibid. 77, 3835 (1996).

[15] M. Sampoli, G. Ruocco, and F. Sette, Phys. Rev. Lett. 79, 1678 (1997), and references therein.

[16] F. Sette, G. Ruocco, M.H. Krisch, C. Masciovecchio, and G. Monaco, Science 280, 1550 (1998).

[17] C. Masciovecchio et al., Phys. Rev. Lett. 80, 544 (1998).

[18] G. Monaco, D. Fioretto, C. Masciovecchio, G. Ruocco, and F. Sette, Phys. Rev. Lett. 82, 1776 (1999).

[19] M. Soltwisch, G. Ruocco, B. Balschun, J. Bosse, V. Mazzacurati, and D. Quitmann, Phys. Rev. E 57, 720 (1998).

[20] M. Soltwisch et al. (unpublished).

[21] W.M. Du et al., Phys. Rev. E 49, 2192 (1994).

[22] R. Di Leonardo et al. (unpublished).

[23] D. Fioretto, L. Comez, G. Socino, L. Verdini, S. Corezzi, and P. A. Rolla, Phys. Rev. E (to be published).

[24] A. Arbe, D. Richter, J. Colmenereo, and B. Farago, Phys. Rev. E 54, 3853 (1996). 
[25] E. Bartsch et al., Phys. Rev. E 52, 738 (1995), and references therein on previous papers of the same group on the same argument.

[26] G. Li, W.M. Du, J. Hernandez, and H.Z. Cummins, Phys. Rev. E 48, 1192 (1993).

[27] M. Fuchs, W. Götze, and A. Latz, Chem. Phys. 149, 185 (1990).

[28] W. van Megen and S.M. Underwood, Phys. Rev. E 49, 4206 (1994).

[29] A. Tölle, H. Schober, J. Wuttke, and F. Fujara, Phys. Rev. E 56, 809 (1997).

[30] M. Fuchs, I. Hofacker, and A. Latz, Phys. Rev. A 45, 898 (1992).

[31] U. Bengtzelius, Phys. Rev. A 34, 5059 (1986).

[32] J. Bosse and J.S. Thakur, Phys. Rev. Lett. 59, 998 (1987).
[33] F. Sciortino, L. Fabbian, S.H. Chen, and P. Tartaglia, Phys. Rev. E 56, 5397 (1997).

[34] Also, at the highest temperature and for the limiting condition $Q=2 \mathrm{~nm}^{-1}$, the characteristic frequency of the structural relaxation $1 /\left(2 \pi \tau_{\alpha}\right)$ is expected to be lower than $0.4 \mathrm{meV}$. This estimation has been obtained from the $\tau(Q)$ behavior obtained by a recent neutron spin-echo investigation of PB [24]. The same condition holds for the secondary relaxation, whose existence has been demostated by Brillouin light scattering measurements $[7,8]$ and which has been attributed to intrachain conformational changes of PB [8]. Starting from the values of $\tau_{s}$ obtained by BLS in the $Q \rightarrow 0$ limit, one obtains $1 /\left(2 \pi \tau_{s}\right)$ $<0.08 \mathrm{eV}$ at $320 \mathrm{~K}$, so that IXS frequencies lie again in the unrelaxed region. 